\title{
Effect of Continuous Antagonistic Muscle Strengthening and Evjenth-Hamberg Stretching on Pulmonary Function of Forward Head Posture Subjects
}

\begin{abstract}
This research was conducted to investigate the effect of continuous antagonistic muscle strengthening exercise and Evjenth-Hamberg stretching on the pulmonary function of university students with abnormal transformation of forward head posture(FHP). The results of study subject to the continuous antagonistic muscle strengthening(CAS) group $(n=10)$ and EvjenthHamberg stretching(EHS) group( $n=10)$ that was conducted 3 times a week for 6 weeks are as follow. FVC, IVC, and MVV all were shown to be significant in the pre - post comparison between the CAS group and EHS group(p<.05), and significant difference was shown for MW between the two groups(p<.05) in which the CAS group showed better effect. Based on the results above, it is considered that combining continuous antagonistic muscle strengthening exercise has better effect on pulmonary function compared to application of only Evjenth-Hamberg stretching.
\end{abstract}

Key words: Antagonist Strengthening, Evjenth-Hamberg Stretching, Forward Head Posture

\author{
Joo Hyun Park \\ Suwon womens University, Suwon, Korea \\ Received : 14 April 2015 \\ Revised : 12 June 2015 \\ Accepted : 17 July 2015
}

Address for correspondence

Joo-Hyun Park, PT, Ph.D

Department of Physical Therapy, Suwon Womens College, 1098, Juseok-ro, Bongdam-eup, Hwaseong-si, Gyeonggi-do, Korea Tel: 82-31-290-8957

E-mail: park33sp@empal.com

※ Funding for this paper was provided by Suwon Women's University in 2015.

\section{INTRODUCTION}

Every person pursues beauty and proper posture is important in physical beauty. That is having a great appearance, but it also has important meaning of physical functionality. Thus, proper posture not only shows exterior beauty, but it also directly·indirectly influences body growth and development, working ability, and exercise ability in which it also has significant value in health aspect(1). Proper posture is the state of aligned core parts of the body such as feet - legs . hip $\cdot$ spine $\cdot$ shoulders $\cdot$ head and shoulders $\cdot \operatorname{mid}-$ dle of hip · back of shin - ankle are aligned starting from the earflap when seen from the side(2). As computers have become popular muscle-bone system disorders are increasing in students and workers who frequently use computers(3). Especially when looking into a monitor for a long period while maintaining still posture, bad posture is caused due to influence of gravity(4). The most representative posture deformation is forward head posture(FHP) in which it is a posture where the center line of the head is in front of the center line of shoulders(5). The neck moment increases when the head is forward positioned in FHP, complementary bending of upper cervical joint and atlanto occipital joint is caused to fix gaze forward to shorten the back muscle of the neck, and the upper cervical is relatively stuck out forward(6). Continuous maintenance of wrong posture causes upper crossed syndrome in which deep flexor such as rhomboid, serratus anterior, and lower trapezius weakens, and pectoralis major, pectoralis minor, upper trapezius, and levator scapularis are shortened $(3,7,8)$ to cause pain to the head, jaw joint, neck bone, back bone, shoulder, and arm(9). Therefore, proper muscle education on shortened and relaxed structure is required(10). Jang(11) reported that incongruity such as FHP makes sufficient lung expansion difficult that pulmonary function are reduced due to reduction of lung capacity and total lung volume.

Because these problems can be solved through improvement of FHP, it has been suggested in several researches that therapeutic approach is 
required by applying strengthening weakened muscles and stretching shortened muscles to align posture $(9,12,13)$.

In this study, the effect of continuous muscle strengthening applied on antagonistic muscles of shortened SCM, upper trapezius, and pectoralis major and Evjenth-Hamberg stretching applied on shortened muscles on pulmonary function is to be to investigated on FHP subjects.

\section{METHODS}

\section{Subjects}

This study is subject to 20 male and female university students attending a university located in Gyeongnam region with disorder in order of FHP according to the New York State posture attitude standard table. Among the subjects, students with shoulder pain or damage medical history, students with orthopedic or neurological disorder in the spine or upper limbs, students with heart or lung disease medical history, and students who did not agree to understand the research contentn and actively participate were excluded.

\section{Methods}

In this study, subjects were randomly assigned to Continuous antagonist strengthening (CAS) group $(\mathrm{n}=10)$ and Evjenthe-Hamberg stretching (EHS) group $(n=10)$ and then intervention was given 3 times a week for a total of 6 weeks. CAS group took antagonist strengthening exercise for 10s after EHS and then took a rest for $5 \mathrm{~s}$, which was repeated 4 times( 4 sets). After 10 sets of exercise, 10-minute rest time was given, which was repeated 3 times (60 minutes in total). EHS group carried out Evjenthe-Hamberg stretching(14). A preliminary inspection was carried out before intervention and post inspection was carried out on the 3rd and 6th week in order to measure changes.

A total of 20 subjects were randomly classified into two groups and explained exactly about how to stretch one day before the date of commencement of research on the premise that they had already understood how to stretch. As warm up before stretching, 5 minute standing jump was carried out, and the stretching was conducted in the order of left and right SCM, upper trapezius, and pectoralis major.

\section{Evjenth-Hamberg Stretching}

\section{Sternocleidomastoid}

Participants took a supine position on the hospital bed and protruded their heads and necks out of their bed with their shoulders placed at the edge of bed. Their shoulders and chests were fixated with belt. At the same time, an experiment stood in the bedhead. Their heads and necks took position so that patients could feel tension in easily shortened muscles. The therapist held subject's head with his/her hand and held subject's mastoid with his/her fingers so that subject's both ears could be placed into therapist's both palms comfortably. Maintaining this posture, the experimenter rotated subject's head completely toward right side and bent it toward left side while towing them simultaneously. Subjects were instructed to say "stop" when they felt a slight tractive sensation right before they felt pain. This point was set as initial stretching posture. In this initial stretching posture, subjects were instructed to apply a strain as if they were pressing an experimenter's right hand and isometric contraction was induced by exerting a balanced force of the same amount in the opposite direction.

The period of isometric contraction was given for 6s. During contraction, they were instructed to count one, two, three, four, five, and six slowly to prevent blood pressure from rising rapidly as Valsalva maneuver phenomenon that may appear during isometric contraction.

The subject relaxed for 2-3s after contraction, whereas the experimenter moved by hand power into more stretched direction. The experimenter had to move to the point where muscles stopped and at this point, maintained for 15-16s. Finally, to strengthen antagonist, the experimenter looked at the right side and had the subject move further in the direction that hairs sticked out. The experimenter resisted this movement and strengthened subject's antagonistic muscle. The time was given for $6 \mathrm{~s}$, and they were instructed to maintain their relaxed state to take a rest for 10s. It was carried out 4 times repeatedly and the stretching time was 160 s in total.

\section{Upper trapezius}

Subjects were instructed to take a lying position with their heads and necks protruded out of beds. Their shoulders and chests were fixated with belts. The experimenter stood at the bedhead. He held subject's back of the head with his right hand, 
supported subject's head with his wrists and arms, and held subject's jaw with his left hand. Maintaining this posture, the experimenter applied a tractive force. Maintaining this traction, the experimenter rotated subject's cervical vertebrae slowly and completely toward the right side and bent toward the left side. He moved his neck simultaneously when subject's head moved. After then, isometric contraction was induced and maintained for $6 \mathrm{~s}$. To strengthen antagonistic muscle, he maintained the location that he held with his hands and pulled subject's chin toward right side. After then, the experimenter had the subject look at the direction that hairs were sticking out and moved hairs further in the direction that hairs were sticking out. The experimenter resisted this movement to strengthen subject's antagonistic muscles. Time of isometric contraction, relaxing method, and overall stretching time are the same as the methods applied for SCM muscle.

\section{Pectoralis Major}

Subject's posture and experimenter's location are the same as the methods applied for SCM muscle. Thoracic cage was fixated into bed using belt. Before carrying out treatment, subject's knee and hip joint were bent to stabilize waist and back and prevent forward bending of lumbar vertebrae.

\section{a. Abdominal part}

The experimenter used his both hands to hold subject's inner side of arm right above his elbow so that subject's both arms could be completely rotated outward and bent at shoulder joint. At this state, the experimenter bent subject's shoulder joint completely and slowly. After then, isometric contraction was induced for $6 \mathrm{~s}$, and to strengthen antagonistic muscles, the subject put his/her hands under arms to hold on the contrary to the hairs sticking out and bent his/her shoulder joint further in the direction of sticking out, and at this posture, the experimenter gave resistance to strengthen the subject's antagonistic muscles. Isometric contraction time, relaxing method, and overall stretching time are the same as the methods applied for SCM muscle.

\section{b. Sternocostal part}

The experimenter used his both hands to hold subject's elbow and lower arms. The experimenter held the subject so that the subject's arms could be bent and rotated outward completely in the loca- tion that exercises are significantly limited between subject's arm $90^{\circ}$ bending and complete bending. In this state, the experimenter bent subject's shoulder joint completely and slowly. After then, isometric contraction was induced for $6 \mathrm{~s}$, and to strengthen antagonistic muscles, the subject put his/her hands under arms to hold on the contrary to the hairs sticking out and bent his/her shoulder joint further in the direction of sticking out, and at this posture, the experimenter gave resistance to strengthen subject's antagonistic muscles. Isometric contraction time, relaxing method, and overall stretching time are the same as the methods applied for SCM muscle.

\section{c. Clavicular part}

The experimenter used his both hands to hold subject's upper arm of elbow joint. The experimenter held the subject so that subject's arms could be bent and rotated outward completely in the state of shoulder joint $90^{\circ}$ bending and elbow joint $90^{\circ}$ bending. In this state, the experimenter opened subject's shoulder joint slowly and completely. After then, isometric contraction was induced for $6 \mathrm{~s}$, and to strengthen antagonistic muscles, the subject put his/her hands under arms to hold on the contrary to the sticking out and bent his/her shoulder joint further to the direction of sticking out, and at this position, the experimenter gave resistance to strengthen subject's antagonistic muscles. Isometric contraction time, relaxing method, and overall stretching time are the same as the methods applied for SCM muscle.

\section{Continuous Antagonist Strengthening Exercise}

After carrying out Evjenthe-Hamberg stretching (EHS) like the above methods, antagonist strengthening exercise was carried out for neck extensor, lower trapezius, and deltoid posterior. Antagonist strengthening exercise was carried out in the same way as the Evjenthe-Hamberg stretching's final method. Cervical extensor and deltoid muscle posterior strengthening exercise were carried out in a prone position, and lower trapezius strengthening exercise was carried out in a sitting posture. In the strengthening exercise time, strengthening exercise was carried out for $10 \mathrm{~s}$ and then rest time was given for $5 \mathrm{~s}$, which was one(1) set: this set was carried out 4 times repeatedly. During one session, a patient carried out 10 sets. After 10 sets, rest time was given for 10 minutes, which was repeated 3 times: 60 minutes in total. 


\section{Measurement of Pulmonary function}

A skilled inspector was invited for accurate inspection and Fitmate(COSMED LTD. Italy) measuring equipment was used. Before preliminary measurement, repetitive education was conducted for the subjects to be aware of the inspection method and measurement was conducted in standing condition. The inspector encouraged subjects to show maximum respiratory ability and nose plugs were used during measurement in which the subjects were educated not to let air escape between the mouth and mouth piece. 3 items of forced vital capacity(FVC), slow or relaxed vital capacity(SVC), and maximal voluntary ventilation(MVV) were measured as pulmonary functions in which the mean value of 3 measurements were used as measurement values. Approximately 1 hour of resting time was in between each item during the test.

\section{Forced Vital Capacity(FVC)}

Repetitive education was conducted for the subjects to be aware of the inspection method before measurement and the subjects wore nose plugs in standing posture. Looking forward sticking the measuring equipment mouthpiece as close as possible to the lips to prevent air escape, subjects waited for the 'start' signal. When the inspector gave the 'start' signal, subjects breathed 3 4 times in normal breathing and performed maximum inhalation and exhaled as fast and as strong as possible so all the air inside the lung was exhausted.

\section{Slow or Relaxed Vital Capacity(SVC)}

Repetitive education was conducted for the subjects to be aware of the inspection method before measurement and the subjects wore nose plugs in standing posture. Looking forward sticking the measuring equipment mouthpiece as close as possible to the lips to prevent air escape, subjects waited for the 'start' signal and the subjects slowly, deeply inhaled maximally and slowly, deeply exhaled lengthily when the inspector gave the 'start' signal. After enough rest, normal breathing was asked with the same method and the subjects were asked to exhale first and then inhale.

\section{Maximal Voluntary Ventilation(MVV)}

Repetitive education was conducted for the subjects to be aware of the inspection method before measurement and the subjects wore nose plugs in standing posture. Looking forward sticking the measuring equipment mouthpiece as close as possible to the lips to prevent air escape, subjects waited for the 'start' signal. When the inspector gave the 'start' signal, subjects repeated maximally deep and fast inhalation and exhalation for approximately $12 \sim 13$ seconds.

\section{Data Analysis}

This study used SPSS/WIN 18.0 statistical program for statistical processing, independent sampling t-test to identify the general characteristics of research subjects, and repeated measures of ANOVA to identify the changes in pulmonary function depending on treatment period of 3 weeks after and before treatment and 6 weeks after and before treatment in each group. The significance level of all data processing was set as $\alpha=.05$.

\section{RESULTS}

\section{General Characteristics of Research Subjects}

The total number of forward head posture subjects who participated in this study was 20: 10 in continuous antagonist strengthening exercise group and 10 in Evjenth-Hamberg stretching group. Of them, there were 6 and 7 males, respectively, and 4 and 3 females, respectively. Their mean age was 23 years old and 22.8 years old, respectively; their height $172 \mathrm{~cm}$ and $174.8 \mathrm{~cm}$, respectively; and their mean weight $62.8 \mathrm{~kg}$ and $67.7 \mathrm{~kg}$, respectively (Table 1).

Table 1. General characteristics of the subjects

\begin{tabular}{ccccc}
\hline & CASG & EHSG & $\mathrm{t}$ & $\mathrm{p}$ \\
\hline \multirow{2}{*}{ Gender } & Male(n=6) & Male(n=7) & 0.447 & 0.660 \\
& Female(n=4) & Female(n=3) & & \\
Age(years) & $23.0 \pm 2.0$ & $22.8 \pm 1.1$ & 0.269 & 0.791 \\
Height(cm) & $172 \pm 5.6$ & $174.8 \pm 6.3$ & -1.054 & 0.306 \\
Weight(kg) & $62.8 \pm 7.8$ & $67.7 \pm 4.9$ & -1.671 & 0.112 \\
\hline CASG : Continuous Antagonist Strengthening Group \\
EHSG : Evjenthe-Hamberg Stretching Group
\end{tabular}

Comparison of Pre $\cdot$ Post Posture Change According to Therapy Period

The comparison of pre $\cdot$ post FVC, IVC, and MW 
between the groups according to therapy period are shown in (Table 2), (Table 3), and (Table 4).

Table 2. Variation of FVC treatment period on each group (unit : $\ell$ )

\begin{tabular}{cccc}
\hline Variation & Pre & 3weeks & 6weeks \\
\hline CASG & $4.15 \pm 0.71$ & $4.39 \pm 0.71$ & $4.71 \pm 0.77$ \\
EHSG & $4.64 \pm 0.98$ & $4.95 \pm 0.96$ & $4.98 \pm 0.99$ \\
\hline
\end{tabular}

Table 3. Variation of NC treatment period on each group

\begin{tabular}{cccc}
\hline Variation & Pre & 3weeks & 6weeks \\
\hline CASG & $3.86 \pm 1.06$ & $4.49 \pm 0.90$ & $4.80 \pm 0.84$ \\
EHSG & $4.70 \pm 0.73$ & $5.21 \pm 0.70$ & $5.00 \pm 0.77$ \\
\hline
\end{tabular}

Table 4. Variation of MW treatment period on each group

\begin{tabular}{cccc}
\hline Variation & Pre & 3weeks & 6weeks \\
\hline CASG & $79.13 \pm 16.10$ & $107.61 \pm 23.44$ & $123.58 \pm 24.91$ \\
EHSG & $123.72 \pm 42.10$ & $135.22 \pm 26.42$ & $137.00 \pm 26.44$ \\
\hline
\end{tabular}

There was statistically significant difference in comparison of pre $\cdot$ post FVC, IVC, and MVV of within the CASG and EHSG groups according to therapy period(p<.05)(Table 8, 9, 10) and there was statistically significant difference according to therapy period and therapy $\operatorname{method}(p<.05)$. As
Also, Mauchly's sphericity test was conducted on FVC, IVC, and MVV (Table 5, 6, 7).

Table 5. Mauchlys test of FVC

\begin{tabular}{ccccc}
\hline $\begin{array}{c}\text { Within } \\
\text { subject effect }\end{array}$ & Mauchlys W & Chi-square & df & $P$ \\
\hline Treatment period & .495 & 11.970 & 2 & .003 \\
\hline
\end{tabular}

Table 6. Mauchlys test of IVC

\begin{tabular}{ccccc}
\hline $\begin{array}{c}\text { Within } \\
\text { subject effect }\end{array}$ & Mauchlys W & Chi-square & df & $P$ \\
\hline Treatment period & .761 & 4.638 & 2 & .098 \\
\hline
\end{tabular}

Table 7. Mauchlys test of MW

\begin{tabular}{ccccc}
\hline $\begin{array}{c}\text { Within } \\
\text { subject effect }\end{array}$ & Mauchlys W & Chi-square & df & $P$ \\
\hline Treatment period & .058 & 48.291 & 2 & .000 \\
\hline
\end{tabular}

result of effect size test on FVC, IVC, and MVV between the groups according to therapy period, MW showed statistically significant difference (p<.05)(Table 13). However, statistically significant difference was not shown in FVC or IVC(Table $11,12)$.

Table 8. Multivariate tests on FVC

\begin{tabular}{ccccccc}
\hline & & Value & $F$ & Hypothesis df & Error df & P \\
\hline FVC & Pillais Trace & .829 & 41.211 & 2.000 & 17.000 & $.000^{*}$ \\
FVC ${ }^{*}$ group & Pillais Trace & .753 & 25.858 & 2.000 & 17.000 & $.000^{*}$ \\
\hline
\end{tabular}

* p<.05

Table 9. Multivariate tests on IVC

\begin{tabular}{ccccccc}
\hline & & Value & $F$ & Hypothesis df & Error df & P \\
\hline IVC & Pillais Trace & .792 & 32.304 & 2.000 & 17.000 & $.000^{*}$ \\
IVC $^{*}$ group & Pillais Trace & .824 & 39.746 & 2.000 & 17.000 & $.000^{*}$ \\
\hline
\end{tabular}

* p<.05 
Table 10. Multivariate tests on MW

\begin{tabular}{ccccccc}
\hline & & Value & F & Hypothesis df & Error df & P \\
\hline \multicolumn{1}{c}{ MVV } & Pillais Trace & .840 & 44.631 & 2.000 & 17.000 & $.000^{*}$ \\
MVV * group & Pillais Trace & .763 & 27.418 & 2.000 & 17.000 & $.000^{*}$ \\
\hline${ }^{*}$ p $<.05$ & & & & & &
\end{tabular}

Table 11. Teat of between-subject effects on FVC

\begin{tabular}{cccccc}
\hline & Type III SS & df & MS & F & P \\
\hline Group & 2.774 & 1 & 2.774 & 1.254 & .277 \\
Error & 422.189 & 18 & 23.455 & & \\
\hline$*$ p $<.05$ & & & & &
\end{tabular}

Table 12. Teat of between-subject effects on NC

\begin{tabular}{cccccc}
\hline & Type III SS & df & MS & F & P \\
\hline Group & 5.180 & 1 & 5.180 & 2.548 & .128 \\
Error & 31.742 & 18 & 1.763 & & \\
\hline${ }^{*}$ p $<.05$ & & & & &
\end{tabular}

Table 13. Teat of between-subject effects on MW

\begin{tabular}{cccccc}
\hline & Type III SS & df & MS & F & P \\
\hline Group & 12217.974 & 1 & 12217.974 & 6.890 & $.017^{*}$ \\
Error & 31.742 & 18 & 1.763 & & \\
\hline${ }^{*}$ p $<.05$ & & & & &
\end{tabular}

\section{DISCUSSION}

Forward head posture(FHP) means the posture of the center line of the head in front of the center line of shoulders(15). Levator scapularis, SCM, upper trapezius, and pectoralis major are shortened due to FHP and muscular weakening is shown in the region below the neck bone, lower cervical and thoracic erector spinae, middle $\cdot$ lower trapezius, and rhomboid $(16,17)$. Improvement of FHP is the key to reducing these problems in which correction exercise on alignment disorder of head regions is the goal of clinical practitioners(18).

Maintenance of proper posture enables maintenance of efficient and smooth breathing, reduces energy consumption of muscles to minimal level when performing movements, and ability to effectively control the body is gained. On the other hand, change that is bad for breathing occurs when posture is improper in which movements are largely influenced to easily feel fatigue and imbalance of movements is caused(19). Respiratory muscles are weakened due to FHP in which abnormal breathing takes place due to changed posture and can lead to chronic respiratory diseases when neglected for a long period. In the comparison in pulmonary functions of $58 \mathrm{FHP}$ subjects classified into breathing exercise group, gradual bearing exercise group, and control group by Jang(11), effective change was observed in the breathing exercise group and gradual bearing exercise group, and most change was shown in the breathing exercise group. In this study, Fitmate(Cosmed, Italy) was used to measure forced vital capacity(FVC), slow or relaxed vital capacity(SVC), and maximal voluntary ventilation(MVV) to look into the change of pulmonary functions through improvement of FHP. As result of the research, significant difference in the pre post comparison between the continuous antagonistic muscle strengthening group and Evjenth-Hamberg stretching group was shown in FVC, FVC per second, maximum exhalation, inhalation lung capacity, and MVV(p<.05) in which therapeutic effect was seen and especially difference between the groups were shown in the MVV(p<.05) that it was known that improvement is more effective when CAS is accompanied. Regarding other variables, statistic significance was not found, but it was known that accompanying CAS was more improved than the EHS group. Regarding pulmonary function, most are researches on pulmonary diseases or researches by direct exercise related to practical breathing. In this study, posture improvement was compared according to stretching method and following pulmonary functions were analyzed in which there was no relation with previous researches. However, it is considered that this study will become basic data on the influence of FHP improvement effect on pulmonary function according to stretching methods. 


\section{CONCLUSION}

In this study, 20 FHP subjects were classified into a CAS group $(n=10)$ and EHS group $(n=10)$ to investigate pulmonary function improvement effect through continuous antagonistic muscle strengthening and Evjenth-Hamberg stretching for 6 weeks in which the following results were gained. Improvement effect of pulmonary function was significant in pre $\cdot$ post comparison in both CAS group and EHS group $(\mathrm{p}<.05)$ and maximal voluntary ventilation(MVV) showed significant difference $(p<.05)$ in which better effect was shown in the CAS group. Based on the results above, it is considered that combining continuous antagonistic muscle strengthening exercise has better effect on pulmonary function compared to application of only Evjenth-Hamberg stretching.

\section{REFERENCES}

1. Lee CY. Postural patterns of daily life of male high school students by positional distortion. Korea National University of Education A Masters degree 2004.

2. Park CS. Effects of newly developed gymnastics on posture, health related physical fitness and the actual condition of life styles in teenagers. Catholic University of Daegu A Doctors degree. 2010

3. Mekhora K, liston CB, \& Nanthavanij S. The effect of ergonomic intervention on discomfort in computer users with tension neck syndrome. Int J Ind Ergo 2000; 26(3): 367-379.

4. Erik Dalton. Strategies to address forward head postures Part 1: Sacrificing compleity for stability. Massage Magazine. 2006.

5. Johnson GM. The correlation between surface measurement of head and neck posture and the anatomic position of the upper cervical vertebrae. Spine. 1998; 23(8): pp.921-927.

6. Cailliet R. Soft tissue pain and disability 3rd ed. Philadelhia PA FA Davis Co 1996.
7. Lee DH. The effects of Balance Exercise and Stretching Exercise on Forward Head Posture. Daegu University A doctors degree 2011.

8. Myers RS. Saunders Manual of Physical Therapy Practice. Philadelphia. WB Saunders Co 1995.

9. Janda V. Muscles and motor control in cervicogenic disorders:assessment and management. In: Grant R, editor. Physical therapy of the cervical and thoracic spine. New York: Churchill Livingstone 1994.

10. Leon C, Craig L, Graeme C, Laurie H, Patrick CP. Muscle energy Techniques: Advanced soft tissue techniques 3rd ed Elsevier Health Sciences 1996.

11. Jang C. The Effects of Breathing Exercise on Forward Head Posture. Daegu University A Doctors degree. 2010

12. Kendall FP, McCreary EK, Provance PG, Rodger MM, Romani WA. Muscles testing and function with posture and pain 5th ed Lippincott Williams \& Winkins 2005.

13. Wright E, Domenech M, Fisher J. Usefulness of posture training for patients with temporomandibular disorders, J American Den Asso 2000; 131(2): 202-210.

14. Evjenth O, Hamberg J. Muscle stretching in manual therapy: A clinical manual Sweden Alfta rehab 1994.

15. Caneiro JP, OSullivan P, Burnett A, Barach A, ONeil D, Tveit O, Olafsdottir K. The influence of different sitting postures on head/neck posture and muscle activity. Manual Therapy. 2010; 15(1): 54-60.

16. Giallonardo LM. Posture Saunders manual of physical therapy practive, Myers R S(ed) Philadelphia WB Saunders Co 1995.

17. Jull G, falla D, Hodges P. Cervical flexor muscle retraining; physiological mechanisms of efficacy. Paper presented at 2nd international conference on movement dysfunction Edingburgh scotland 2005.

18. Darling D, Kraus S, Glasheen-Wray M. Relationship of head posture and rest position of mandible, J Prosthe Den, 1984; 52(1): 111-115.

19. Lim HS. A Study on Breathing Type in Breath Structure of Korean Dance, The Korean Journal of Dance 2003; 35(1): 139-152. 